\title{
Tratamento de manutenção da asma persistente à admissão em ambulatório de pneumologia pediátrica
}

\author{
Use of asthma controller drugs at admission \\ to a pediatric pulmonology outpatient clinic
}

\author{
Paulo A.M. Camargos 1 , Sandra C. Profeta ${ }^{2}$
}

\section{Resumo}

Objetivo: a corticoterapia inalatória é amplamente empregada nos países desenvolvidos, mas, dado ao seu elevado custo, tem sido usada de forma limitada nos países em desenvolvimento. O presente estudo teve como objetivo a quantificação do uso de medicação profilática em crianças com asma persistente moderada ou grave, quando de sua admissão em ambulatório especializado.

Métodos: trata-se de um estudo descritivo, baseado nas informações disponíveis nos prontuários de 560 crianças entre quatro e catorze anos, selecionadas aleatoriamente e atendidas entre abril de 1996 a dezembro de 2000, no ambulatório de pneumologia pediátrica do Centro Geral de Pediatria/Fundação Hospitalar do Estado de Minas Gerais.

Resultados: dos 560 pacientes, $61,8 \%$ eram do sexo masculino, $69,5 \%$ tinham entre quatro e nove anos; a primeira crise ocorreu antes de um ano de idade em $55,8 \%$, e 70,5\% deles apresentavam asma persistente moderada. A internação nos últimos doze meses ocorreu em $42,7 \%$ das crianças, e o atendimento em serviços de urgência, em $92,7 \%$. O percentual de pacientes em tratamento de manutenção foi de $27,3 \%$, e da corticoterapia inalatória, $17,1 \%$, para os residentes na região metropolitana de Belo Horizonte. Observouse redução da profilaxia com corticoterapia sistêmica de $14,3 \%$ para $4,2 \%$ entre 1996 e 2000.

Conclusões: os índices de corticoterapia inalatória situaram-se em níveis superiores aos observados em estudos brasileiros e comparáveis a alguns internacionais, ocorrendo paralelamente uma redução do uso de corticoterapia sistêmica de manutenção. Estes resultados podem estar associados à implantação do programa de reorganização da assistência pública à criança asmática em Belo Horizonte.

J Pediatr (Rio J) 2003;79(3):233-8: asma, antiasmáticos, prevenção e controle.

\begin{abstract}
Objective: inhaled corticosteroids are widely used in developed countries. On the other hand, due to its high cost, this kind of drug is underused in asthmatic patients living in developing countries. The present study aims at verifying the use of controller medication in children with moderate or severe persistent asthma at admission to a specialized outpatient facility.
\end{abstract}

Methods: descriptive study with 560 children aged 4 to 14 , with diagnosed asthma, who were admitted between April 1996 and December 2000. The patients were randomly selected.

Results: of 560 patients, $61.8 \%$ were male; $69.5 \%$ were between 4 and 9 years old. The first acute attack occurred before the first year of life in $55.8 \%$ of the cases, and $70.5 \%$ had physician-diagnosed persistent moderate asthma. In the previous 12 months, $42.7 \%$ of the children had been admitted to hospital and $92.7 \%$ were assisted in emergency rooms. Considering those living in the metropolitan area of Belo Horizonte, the average use of some kind of controller drug was $27.3 \%$, and $17.1 \%$ of inhaled corticosteroids. The use of oral corticosteroids decreased from $14.3 \%$ in 1996 to $4.2 \%$ in 2000.

Conclusions: the rate of inhaled corticosteroid therapy was higher than those found in Brazilian studies and it is comparable to the results of some international studies. A decrease in the use of oral corticosteroids was also observed. Both findings may be related to the implementation of an asthma program in the public health system of Belo Horizonte, which began in 1996.

JPediatr (RioJ) 2003;79(3):233-8: asthma, antiasthmatic agents, prevention and control.

1. Professor Titular Doutor, Departamento de Pediatria da Faculdade de Medicina. Chefe da Unidade de Pneumologia Pediátrica do Hospital das Clínicas, Universidade Federal de Minas Gerais (UFMG).

2. Preceptora do Programa de Residência Médica e membro do Grupo de Pneumologia Pediátrica, Centro Geral de Pediatria, Fundação Hospitalar do Estado de Minas Gerais. Mestre em Medicina (Pediatria) pela UFMG.

Artigo submetido em 23.10.02, aceito em 12.03.03. 


\section{Introdução}

A mudança do enfoque terapêutico da asma, a partir dos avanços no conhecimento da sua fisiopatologia, ocorreu nos últimos 20 anos. Sabe-se hoje que a inflamação é o evento fisiopatológico básico, e a indicação da medicação antiinflamatória inalada na asma persistente é consensual, possibilitando controle efetivo da sua morbidade ${ }^{1-4}$. O grande esforço na divulgação dos conhecimentos e padronização das condutas, através de consensos internacionais e nacionais, tem sido frustrado, e estudos evidenciam que a asma continua sendo indevidamente diagnosticada e trata$\mathrm{da}^{5-9}$. A distância entre o conhecimento adquirido e sua aplicação ainda é grande, mesmo em países com elevados índices de profilaxia ${ }^{10-12}$.

No Brasil, a asma constitui a terceira causa de hospitalização entre crianças e adultos jovens, gerando expressivos custos financeiros para o sistema de saúde e elevado custo individual, com repercussões no absenteísmo escolar e no trabalho, baixo rendimento físico e pelos impactos psicossociais ${ }^{2}$. Os estudos brasileiros revelam uma reduzida proporção de tratamento com corticoterapia inalatória, variando de zero, em estudo piloto realizado em Belo Horizonte, no período de 1994 a $1995^{13}$, a 6,0\%, na observação de Cabral e colaboradores, em $1998^{7}$. Infelizmente, a revisão bibliográfica realizada pelos autores não identificou nenhum estudo sobre este tema em outros países latino-americanos.

O presente estudo objetivou avaliar e quantificar a proporção de uso do tratamento de manutenção na asma persistente, previamente à admissão do paciente em um ambulatório especializado.

\section{Métodos}

\section{Local, período de realização e população estudada}

$\mathrm{O}$ estudo foi realizado no serviço de pneumologia e alergia pediátrica do Centro Geral de Pediatria (CGP), pertencente à Fundação Hospitalar do Estado de Minas Gerais (FHEMIG), no período de 04/1996 a 31/12/2000. A clientela é composta, basicamente, por crianças assistidas pelo Sistema Único de Saúde (SUS). Entre os 1.050 pacientes elegíveis para o estudo, foram selecionados, de forma aleatória simples, 560 pacientes de primeira consulta.

\section{Dinâmica do serviço}

O Centro Geral de Pediatria (CGP) é um serviço de referência vinculado à Secretaria Estadual de Saúde, que dispensa atendimentos de urgência, emergência, de pediatria clínica geral e subespecialidades. O setor de urgência atende, aproximadamente, 300 pacientes/dia, a unidade de internação dispõe de 142 leitos, e o CTI de 12 leitos.

$\mathrm{O}$ ambulatório de pneumologia e alergologia pediátrica integra a unidade de cuidados secundários e não tem vinculação específica com as unidades da rede ambulatorial operada pela administração municipal, que pertencem ao programa de asma da Prefeitura de Belo Horizonte. Os pacientes nele atendidos são procedentes do setor de urgência e da unidade de internação do próprio CGP e da demanda espontânea da clientela residente na RMBH.

Compõem a equipe clínica, pediatras com formação em pneumologia e alergologia e médicos-residentes em fase de formação nestas subespecialidades, que se servem de protocolos e fichas de atendimento padronizadas. Os critérios contidos no documento intitulado Global Initiative for Asthma (GINA) ${ }^{2}$ são adotados pela equipe, para assegurar a homogeneidade do diagnóstico e classificação da gravidade da asma.

\section{Definições operacionais}

\section{Conceito e classificação da asma}

O diagnóstico e classificação da asma foram baseados em critérios clínicos e funcionais $1,2,14 \mathrm{e}$, muito especialmente, nas recomendações contidas no GINA ${ }^{2}$, no qual se prevê que a presença de um parâmetro de gravidade é suficiente para classificar o paciente em uma dada categoria.

Utilizou-se ainda os valores de referência do pico do fluxo expiratório obtidos por Godfrey ${ }^{15}$, e considerou-se $80,0 \%$ do valor previsto como limite inferior da normalidade (Tabela 1).

\section{Medicação profilática}

Foram considerados medicamentos profiláticos as drogas citadas no consenso brasileiro e no GINA ${ }^{1,2}$, a saber, teofilina de liberação lenta, beta2-agonista de ação duradoura, cetotifeno, corticosteróides orais, corticosteróides inalados, nedocromil, cromoglicato e antileucotrienos. Avaliou-se a utilização de medicação sistêmica ou inalatória, de uso regular no período intercrise, previamente à primeira consulta no serviço, e o tempo de utilização superior a trinta dias.

\section{Critérios de inclusão e exclusão}

Foram incluídos no estudo pacientes de quatro a catorze anos, no primeiro atendimento no serviço, residentes na região metropolitana de Belo Horizonte, com diagnóstico de asma persistente moderada ou grave. Foram excluídos os pacientes menores de quatro anos, aqueles com outras pneumopatias crônicas e malformações pulmonares congênitas ou adquiridas. Os prontuários com informações insuficientes foram substituídos pelo número de ordem mais próximo da seqüência aleatória.

\section{Aspectos estatísticos}

Um protocolo elaborado especificamente para o estudo foi preenchido com dados selecionados a partir dos prontuários. 
Tabela 1 - Classificação da gravidade da asma

\begin{tabular}{|c|c|c|c|c|}
\hline & $\begin{array}{c}\text { Nível } 1 \\
\text { Intermitente }\end{array}$ & $\begin{array}{c}\text { Nível } 2 \\
\text { Persistente leve }\end{array}$ & $\begin{array}{c}\text { Nível } 3 \\
\text { Persistente moderada }\end{array}$ & $\begin{array}{c}\text { Nível } 4 \\
\text { Persistente grave }\end{array}$ \\
\hline Sintomas & $\begin{array}{l}\text { Menos que uma vez } \\
\text { por semana }\end{array}$ & $\begin{array}{l}\text { Mais que uma vez } \\
\text { por semana e menos } \\
\text { que uma vez ao dia }\end{array}$ & Diários & Diários \\
\hline Exacerbações & $\begin{array}{c}\text { De curta duração } \\
\text { e leves }\end{array}$ & $\begin{array}{l}\text { Podem afetar } \\
\text { atividade física e sono }\end{array}$ & $\begin{array}{l}\text { Podem afetar } \\
\text { atividade física e sono }\end{array}$ & Freqüentes \\
\hline Sintomas noturnos & $\begin{array}{c}\text { Menos que } \\
\text { duas vezes ao mês }\end{array}$ & $\begin{array}{c}\text { Mais que } \\
\text { duas vezes ao mês }\end{array}$ & $\begin{array}{c}\text { Mais que } \\
\text { uma vez por semana }\end{array}$ & Freqüentes \\
\hline $\mathrm{VEF}_{1}$ ou PFE* & $\geq 80 \%$ & $\geq 80 \%$ & $60-80 \%$ & $<60 \%$ \\
\hline Variabilidade do PFE & $<20 \%$ & $20-30 \%$ & $>30 \%$ & $>30 \%$ \\
\hline
\end{tabular}

${ }^{*} V_{E F}$ : volume expiratório forçado no primeiro segundo, PFE: pico do fluxo expiratório.

O cálculo do tamanho amostral foi baseado nos seguintes parâmetros: número de pacientes elegíveis (total de 1.050 registros), estimativa média de uso de $25,0 \%$ por ano para a corticoterapia inalatória, erro alfa de $5,0 \%$, perda de $20,0 \%$ e margem de erro de mais ou menos $2,0 \%$ para a proporção de uso de drogas profiláticas encontrada a cada ano. O número de pacientes necessários nos anos de 1996, 1997, 1998, 1999 e 2000 foi, respectivamente, 35, 115, 115,153 e 142. Foram calculadas as distribuições de freqüência, médias, medianas e qui-quadrado de tendência linear. A variação de proporção de uso de corticosteróide inalatório foi expressa pelo cálculo dos limites inferior e superior do intervalo de confiança a 95,0\%.

\section{Aspectos éticos}

O projeto foi aprovado pelo Comitê de Ética em Pesquisa do Centro Geral de Pediatria e da Universidade Federal de Minas Gerais.

\section{Resultados}

\section{Características descritivas}

As características gerais da população estudada encontram-se na Tabela 2.

Dos 560 pacientes, $61,8 \%$ eram do sexo masculino, correspondendo a uma relação de 1,6/1. A faixa etária das crianças admitidas no estudo mostrou predomínio entre quatro e nove anos, correspondendo a $69,8 \%$, com mediana equivalente a 85 meses. Os pacientes residentes em Belo Horizonte representaram 60,2\% do total, e os demais foram procedentes de outros municípios da região metropolitana. Observou-se que a maioria dos pais exercia atividades braçais na construção civil $(40,2 \%)$, em serviços autôno$\operatorname{mos}(7,3 \%)$, ou eram aposentados $(2,9 \%)$. Quanto às mães, exerciam atividades como empregadas domésticas $(20,2 \%)$, serviços autônomos $(10,4 \%)$ e donas de casa $(45,5 \%)$.
Tabela 2 - Características descritivas da população estudada $(n=560)$

\begin{tabular}{|c|c|c|}
\hline Variável & $\mathbf{n}$ & $\%$ \\
\hline \multicolumn{3}{|l|}{ Sexo } \\
\hline Masculino & 346 & 61,8 \\
\hline Feminino & 214 & 38,2 \\
\hline \multicolumn{3}{|c|}{ Faixa etária à $1^{\mathrm{a}}$ crise (anos) } \\
\hline menor de 1 & 313 & 55,9 \\
\hline $1-2$ & 104 & 18,6 \\
\hline $2-4$ & 78 & 13,9 \\
\hline$>4$ & 51 & 9,1 \\
\hline Sem informação & 14 & 2,5 \\
\hline \multicolumn{3}{|c|}{$\begin{array}{l}\text { Faixa etária ao ingresso } \\
\text { no ambulatório (anos) }\end{array}$} \\
\hline $4-6$ & 195 & 34,8 \\
\hline $7-9$ & 196 & 35,0 \\
\hline $10-14$ & 169 & 30,2 \\
\hline \multicolumn{3}{|l|}{ Município de origem } \\
\hline Belo Horizonte & 337 & 60,2 \\
\hline Outros & 223 & 39,8 \\
\hline Classe social - pai & 377 & 67,3 \\
\hline Subproletariado* & 190 & 50,4 \\
\hline Proletariado $^{\dagger}$ & 145 & 38,5 \\
\hline Burguesia $¥$ & 42 & 11,1 \\
\hline Classe social - mãe & 356 & 63,6 \\
\hline Subproletariado* & 271 & 76,1 \\
\hline Proletariado ${ }^{\dagger}$ & 64 & 18,0 \\
\hline Burguesia ${ }^{\ddagger}$ & 20 & 5,9 \\
\hline
\end{tabular}

Trabalhadores braçais assalariados, autônomos sem negócio próprio, donas de casa.

† Trabalhadores de serviços assalariados, funcionários públicos, aposentados.

‡ Profissionais de nível técnico.

\section{Características clínico-funcionais}

As variáveis estudadas para caracterização clínico-funcional da asma são apresentadas na Tabela 3. 
Tabela 3 - Características clínico-funcionais da asma na população estudada $(n=560)$

\begin{tabular}{|c|c|c|}
\hline Variável & $\mathbf{n}$ & $\%$ \\
\hline \multicolumn{3}{|l|}{ Periodicidade dos sintomas } \\
\hline Menor que $1 \mathrm{vez} /$ semana & 398 & 71,1 \\
\hline $1 \mathrm{vez}$ ou mais / semana & 54 & 9,6 \\
\hline Diário & 12 & 2,1 \\
\hline Contínuo com exacerbações freqüentes & 69 & 12,6 \\
\hline Sem informação & 27 & 4,8 \\
\hline \multicolumn{3}{|l|}{ Sintomas noturnos } \\
\hline Menor que 2 vezes / mês & 26 & 4,6 \\
\hline Maior que 2 vezes / mês & 140 & 25,0 \\
\hline Maior que $1 \mathrm{vez} /$ semana & 74 & 13,2 \\
\hline Freqüentes & 284 & 50,7 \\
\hline Não informa & 36 & 6,4 \\
\hline \multicolumn{3}{|l|}{ Atividade física } \\
\hline Normal & 17 & 3,0 \\
\hline Afetada ou não nas exacerbações & 6 & 1,1 \\
\hline Afetada nas exacerbações & 102 & 18,2 \\
\hline Limitação contínua & 337 & 60,2 \\
\hline Não relatada & 98 & 17,5 \\
\hline \multicolumn{3}{|l|}{ Absenteísmo escolar } \\
\hline Sim & 269 & 48,0 \\
\hline Não & 75 & 13,4 \\
\hline Não freqüenta escola & 216 & 38,6 \\
\hline \multicolumn{3}{|l|}{ Gravidade da asma } \\
\hline Moderada & 395 & 70,5 \\
\hline Grave & 165 & 29,5 \\
\hline \multicolumn{3}{|l|}{ Atendimento prévio na urgência } \\
\hline Sim & 520 & 92,9 \\
\hline Não & 23 & 4,1 \\
\hline Não informa & 17 & 3,0 \\
\hline \multicolumn{3}{|l|}{ Hospitalização prévia } \\
\hline Sim & 239 & 42,7 \\
\hline Não & 253 & 45,2 \\
\hline Sem informação & 68 & 12,1 \\
\hline \multicolumn{3}{|l|}{ Medidas do pico do fluxo expiratório } \\
\hline Igual ou maior que $80 \% *$ & 111 & 19,8 \\
\hline Menor que $80 \%$ igual ou maior que $60 \%$ * & 105 & 18,7 \\
\hline Menor que $60 \%{ }^{*}$ & 53 & 9,5 \\
\hline Não realizado ${ }^{\dagger}$ & 291 & 52,0 \\
\hline
\end{tabular}

* do valor previsto.

$†$ não realizado por limitações técnicas.

A freqüência das crises foi menor que uma vez por semana para $72,0 \%$ das crianças. Sintomas noturnos estiveram presentes em $50,7 \%$ dos casos, e atividade física limitada em $60,2 \%$. O absenteísmo escolar foi de $48,0 \%$ entre as 212 crianças que frequientavam a escola. Quanto à classificação da gravidade, $70,5 \%$ dos pacientes apresentavam quadros moderados, e 29,5\% quadros graves. Observou-se, ainda, que $92,0 \%$ das crianças recorreram ao atendimento de urgência nos doze meses prévios à admissão no ambulatório especializado, e 42,7\% haviam sido hospitalizados neste mesmo período. A medida do pico do fluxo expiratório foi possível em 269 crianças, e entre estas, $58,7 \%$ apresentavam valores inferiores a $80,0 \%$ do predito.

\section{Uso da medicação antiinflamatória na asma}

A Figura 1 documenta a utilização de medicação profilática na população estudada.

Observou-se que o uso de algum tipo de medicação de manutenção prévia, isolada ou em associação, foi de $25,7 \%$ em 1996; 33,9\% em 1997; 26,1\% em 1998; 25,9\% em 1999; e 25,4\% em 2000, com média de 27,3\% (IC 95\%, $23,7 \%$ a $31,3 \%$ ). Estabelecendo-se um paralelo entre a proporção de uso de beclometasona e prednisona oral em dias alternados, em cada ano de estudo, foram observados índices oscilantes, sem significância estatística $(\mathrm{p}=0,84)$, de corticoterapia inalatória de $14,3 \%, 21,7 \%, 13,0 \%, 17,1 \%$, $17,6 \%$ com média de $17,1 \%$ (IC $95 \%, 14,2 \%$ a $20,6 \%$ ) e tendência decrescente $(\mathrm{p}=0,04)$ para o uso de prednisona de $14,3 \%, 10,4 \%, 7,0 \%, 7,8 \%, 4,8 \%$, para os anos de 1996 , 1997, 1998, 1999 e 2000, respectivamente, com média de 7,7\% (IC 95\%, 5,7\% a 10,3\%).

A prescrição prévia de teofilina de liberação lenta e de cromoglicato foi muito reduzida, e o cetotifeno teve seu pico de uso em 1998 (7,0\%) e valores decrescentes, inferiores a 5,0\%, nos demais períodos. Não se identificou nenhum paciente em uso de moduladores de leucotrienos.

\section{Discussão}

Os avanços obtidos no tratamento da asma a partir da introdução da corticoterapia inalatória no tratamento da asma persistente é evidenciado através da redução da hospitalização, dos atendimentos de urgência, do absenteísmo escolar, da gravidade e da evolução ${ }^{7,16-19}$.

O presente estudo permite supor que, mesmo com as limitações existentes nos serviços públicos de saúde, inclusive a falta de integração entre os diferentes níveis e esferas de atenção, é possível implantar ações que objetivem a introdução de corticoterapia inalatória na rede pública, de que é exemplo o programa de reorganização da assistência pública à criança asmática de Belo Horizonte. Este progra-

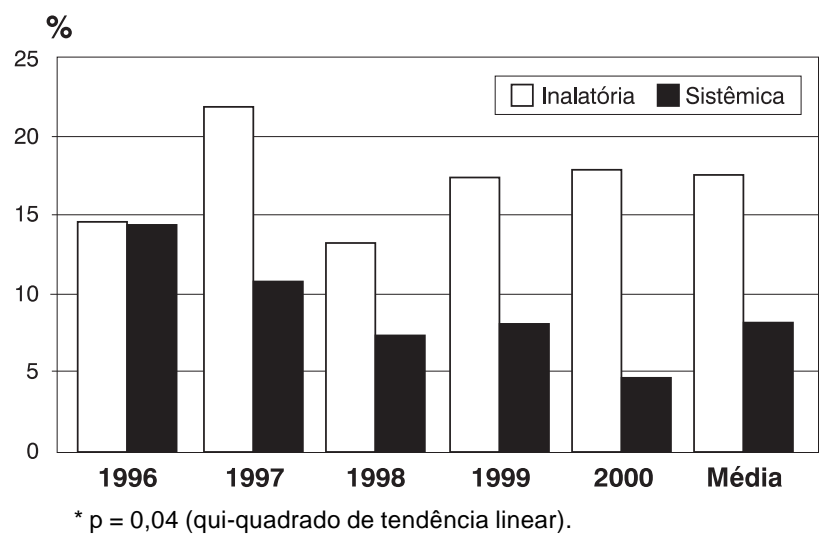

Figura 1 - Uso de corticoterapia sistêmica e inalatória à admissão* 
ma teve início informal em 1996 e implantação gradual a partir de 1997, pela Secretaria Municipal de Saúde em parceria com o Departamento de Pediatria da Universidade Federal de Minas Gerais, e teve como eixos o treinamento dos pediatras dos centros de saúde e a distribuição gratuita de beclometasona e salbutamol sob a forma de spray e espaçadores valvulados. O programa prioriza a faixa etária de maior morbidade, ou seja, os menores de cinco anos, que representam cerca de $75 \%$ da clientela infanto-juvenil beneficiada pelo Programa.

Na população estudada, a distribuição por sexo mostra uma relação de 1,6/1 para o sexo masculino, concordante com dados da literatura, que apontam um predomínio do sexo masculino até a adolescência ${ }^{20}$, e concordante com o estudo nacional realizado por Duarte e Camargos ${ }^{8}$. Observou-se que a mediana de idade das crianças admitidas foi de 86 meses, apesar de o início das crises ter ocorrido em $55,8 \%$ das crianças antes de 12 meses de vida, terem $92,9 \%$ delas sido atendidas em serviços de urgência, e 42,7\% terem necessitado internação prévia, resultado que se aproxima daqueles obtidos por Cabral e colaboradores ${ }^{7}$, que foram de $98,0 \%$ e $82,0 \%$, respectivamente.

Para que se possa estabelecer uma análise comparativa entre este e os vários estudos sobre o uso de medicamentos inalatórios, deve-se atentar para as diferenças metodológicas entre eles, tais como, variações quanto ao objetivo principal do estudo, faixa etária estudada, classificação da gravidade da asma, tipo de droga analisada e período de realização do estudo.

Países europeus, como a Finlândia, Islândia, Holanda, Noruega e Suécia, apresentam os mais elevados índices de uso de costicosteróides inalatórios observados na década de $90(80,0 \%, 62,0 \%, 58,0 \%, 45,9 \%$ e $45,1 \%)$, entretanto, os estudos referem-se à população geral e não consideram a gravidade da asma ${ }^{10,21,22}$. Embora possuam padrão socioeconômico-cultural e um sistema de saúde de elevado nível, priorizando a prevenção, outros países europeus apresentam menor proporção de pacientes asmáticos em uso destes medicamentos, como a Alemanha $(30,8 \%)$ e a Eslováquia $(39,2 \%)^{23}$. Estudando 555 crianças de escolas primárias públicas de Paris, em 1994, Momas ${ }^{5}$ chamou atenção para o fato de a asma ainda ser indevidamente diagnosticada e tratada, ao detectar índice de $14,3 \%$ de profilaxia, e o uso de corticoterapia inalatória limitar-se a $6,3 \%$. Estes estudos não consideraram a gravidade da asma, o que limita a comparação de índices com o atual estudo.

Pesquisas realizadas nos Estados Unidos alertam para as grandes variações de uso de medicação profilática inalada. Uma avaliação em três diferentes regiões, Boston (Massachussets), Rochester (New York) e New Haven (Connecticut), no período de 1988 a 1990, em crianças com asma de gravidade não definida, evidenciou índices de uso de cromoglicato e corticosteróide inalatório de $11,0 \%$, $19,0 \%$ e $33,0 \%{ }^{18}$. Outro estudo, aglomerando oito locais do centro de sete grandes metrópoles dos Estados Unidos, realizado entre 1992 e 1993, por Crain ${ }^{24}$, observou um índice médio de 47,5\% de antiinflamatório inalado na asma grave. As proporções de uso da terapia de manutenção da asma nos estudos mencionados não são comparáveis aos encontrados no presente estudo, pelas diferentes características das populações estudadas.

Um projeto implantado na República Tcheca, com o objetivo de coordenar uma estratégia para abordagem da asma $^{25}$, foi iniciado em 1997 com o treinamento dos profissionais de atendimento primário, representando $53,8 \%$ dos pediatras e $26,3 \%$ dos clínicos gerais. Foram então comparados os esquemas terapêuticos prescritos em 1993 com a situação pós-treinamento de 1999, que mostrou decréscimo no uso de cetotifeno $(44,0 \%$ para $19,0 \%)$, de cromoglicato $(24,0 \%$ para $5,0 \%)$, de xantinas $(25,0 \%$ para $4,0 \%)$, e também o aumento na prescrição de corticosteróide inalatório de $3,0 \%$ para $52,0 \%$. Respeitadas as diferenças metodológicas, tais como o estudo ter sido realizado avaliandose a população geral, a utilização de registros de prescrições e a não consideração da gravidade clínica, o presente estudo revela também tendência de redução da proporção de uso de prednisona como profilático e baixos índices de uso de cromoglicato, xantinas e cetotifeno.

No Brasil, estudo realizado no Ceará ${ }^{9}$, com 960 crianças, das quais $23,0 \%$ apresentavam asma persistente moderada ou grave, revelou que apenas $1,5 \%$ das crianças fazia uso de beclometasona, e 5,2\% de cromoglicato ou nedocromil. Proporções semelhantes, $6,1 \%$ de corticosteróide inalatório, foram encontradas por McGill e colaboradores ${ }^{6} \mathrm{em}$ Wisconsin, entre 1992 e 1996, em estudo realizado em crianças carentes de três a cinco anos. Estes índices são inferiores àqueles encontrados no presente estudo, ou seja, 17,1\%, em média, para corticosteróide inalatório. Em Juiz de Fora ${ }^{8}$, encontrou-se índices de 4,6\% de uso de antiinflamatório inalado, sendo a corticoterapia inalatória utilizada em apenas $0,8 \%$ dos pacientes com asma persistente moderada e grave. Índices de profilaxia inalada, também inferiores aos observados em Belo Horizonte, foram encontrados por Cabral e colaboradores ${ }^{7}$ no município de São Paulo, que observou o uso de corticoterapia inalatória em 6,0\% dos pacientes. Considerando-se os dados de estudos internacionais e nacionais, a profilaxia inalada, no presente estudo, encontra-se numa faixa intermediária. Entretanto, os índices de cobertura ainda são baixos, mostrando que o programa de controle da asma implantado em Belo Horizonte precisa ampliar a cobertura aos maiores de cinco anos.

Apesar das características socioeconômicas e culturais da população estudada e das limitações do programa da asma, observa-se que o índice de uso de medicação de manutenção situou-se em torno de $17 \%$ ao longo dos cinco anos do estudo, a despeito de variações anuais, tal como a que o presente estudo detectou no período 1997-1998. Informações extra-oficiais obtidas pelos autores revelaram a ocorrência de um abastecimento irregular de corticóide inalatório no biênio, por razões de ordem operacional e administrativa. 
A partir da implantação do projeto, o treinamento dos pediatras que atuam nos centros de saúde operados pela Prefeitura Municipal, que também estão vinculados direta ou indiretamente a outros serviços de urgência e emergência da região metropolitana de Belo Horizonte, entre eles o CGP, pode ter contribuído para a obtenção dos índices encontrados e para a mudança no perfil do tratamento, como a consistente e progressiva redução do uso de corticoterapia sistêmica. Entretanto, limitações, provavelmente relacionadas com o protocolo usado no presente estudo, não permitiram aos autores interpretações mais detalhadas sobre a relativa desproporcionalidade entre o decréscimo e a elevação do uso da via sistêmica e inalatória, respectivamente.

Constata-se então que, mesmo em países em via de desenvolvimento, nos quais são por demais reconhecidas as deficiências do serviço público de saúde, é possível alcançar índices minimamente satisfatórios de cobertura para o tratamento de manutenção da asma. O treinamento contínuo do pessoal de saúde, a maior integração entre as unidades de atendimento primário e terciário, para assegurar a continuidade do tratamento após alta hospitalar, a ênfase nos programas educativos e de automanejo, associado ao fornecimento gratuito e regular de corticosteróide inalatório, são elementos fundamentais para que o conhecimento teórico divulgado pelos consensos atinja seu objetivo prático.

\section{Agradecimentos}

Os autores agradecem aos Profs. Dirceu Solé, da Universidade Federal de São Paulo, e Joaquim Antonio César Mota, da Universidade Federal de Minas Gerais, pelas críticas e sugestões apresentadas à versão original deste artigo.

\section{Referências bibliográficas}

1. Sociedade Brasileira de Pneumologia e Tisiologia, Sociedade Brasileira de Alergia Imunopatologia, Sociedade Brasileira de Pediatria. II Consenso Brasileiro no Manejo da Asma. Revista Brasileira de Alergia e Imunopatologia 1998;21 Supl 1:171-276.

2. NHLBI/WHO Workshop Report/Global strategy for asthma management and prevention. Bethesda (MD): NIH; 1995. Publication $\mathrm{N}^{\circ} 02-3659$.

3. Van Essen-Zandvliet EE, Hughes MD, Waalkens HJ, Duiverman EJ, Pocock SJ, Kerrebijn KF. Effects of 22 months of treatment with inhaled corticosteroids and/or beta 2 agonist on lung function, airway responsiveness, and symptoms in children with asthma. Am Rev Respir Dis 1992;146:547-54.

4. O'Byrne PM, Pedersen S. Measuring efficacy and safety of different inhaled corticosteroid preparations. J Allergy Clin Immunol 1998; 102:879-86.

5. Momas I, Dartiguenave C, Fauroux B, Bourdais M, Poinsard R, Grimfeld A, et al. Prevalence of asthma or respiratory symptoms among children attending primary schools in Paris. Pediatr Pulmonol 1998;26:106-12.

6. McGill KA, Sorkness CA, Ferguson-Page C, Gern JE, Havighurst TC, Knipfer B, et al. Asthma in non-inner city head start children. Pediatrics 1998;102:77-83.
7. Cabral AL, Carvalho WA, Chinen M, Barbiroto RM, Boueri FM, Martins MA. Are International asthma guidelines effective for lowincome Brazilian Children with asthma? Eur Respir J 1998;12:35-40.

8. Duarte M, Camargos P. Emprego de medicação profilática na asma persistente. J Pediatr (Rio J) 1999;75:456-62.

9. Holanda MA, Amaral JJF, Freitas O, Silva MMM, Barbosa AT, Catunda LG, et al. Perfil do uso de medicamentos em crianças com asma em unidades de saúde em Fortaleza-Ceará. J Pneumologia 1998;24:S19.

10. Lagerlov P, Veninga CC, Muskova M, Hummers-Pradier E, Stalsby Lundborg S, Andrew M, et al. Asthma management in five European countries: doctors' knowledge, attitudes and prescribing behavior. Eur Respir J 2000;15:25-29.

11. Halterman JS, Aligne A, Auinger P, McBride JT, Szilagyi PG. Inadequate therapy for asthma among children in the United States. Pediatrics 2000;105:272-6.

12. Ordoñez GA, Phelan PD, Olinsky A, Robertson CF. Preventable factors in hospital admissions for asthma. Arch Dis Child 1998;78:143-7.

13. Lasmar LMLBF, Fontes MJF, Guerra HL, Jentzsch NS. Perfil da assistência pública à criança e ao adolescente asmático. RMMG 2000;10(4):208-12.

14. Warner JO, Naspitz CK. Third International Pediatric Consensus Statement on the Management of childhood Asthma. Pediatr Pulmonol 1998;25:1-17.

15. Godfrey S, Kamburoff PL, Nairn JR. Spirometry lung volumes and airway resistance in normal children aged 5 to 18 years. Br J Dis Chest 1970;64:15-24.

16. Lewis MA, Rachelefsky G, Lewis CE, Leake B, Richards W. The termination of a randomized clinical trial for poor Hispanic children. Arch Pediatr Adolesc Med 1994;148:364-7.

17. Eggleston PA, Malveaux FJ, Butz AM, Huss K, Thompson L, Kolodner K, et al. Medications used by children with asthma living in the inner city. Pediatrics 1998;101:349-54.

18. Homer CJ, Szilagyi P, Rodewald L, Bloom SR, Greenspan P, Yazdgerdi S, et al. Does quality of care affect rates of hospitalization for childhood asthma? Pediatrics 1996;98:18-23.

19. Suissa S, Ernst P, Benayoun S, Baltzan M, Cai B. Low-dose inhaled corticosteroids and the prevention of death from asthma. N Eng J Med 2000;343:332-6.

20. Carr W, Zeitel L, Weiss K. Variations in asthma hospitalizations and deaths in New York City. Am J Public Health 1992;82:59-65.

21. Korppi M, Kuikka L, Remes K. Preventive therapy for asthma in children: a 9-year experience in Eastern Finland. Eur Respir J 1995; 8:1318-20.

22. Gislason T, Oleafsson O, Sigfvaldason A. Users of antiasthma drugs in Iceland: a drug utilization study. Eur Respir J 1997;10(6):1230-4.

23. Veninga CC, Lagerlov P, Wahlstrom R, Muskova M, Denig P, Berkhof $\mathrm{J}$, et al. Evaluating an educational intervention to improve the treatment of asthma in four European Countries. Am J Respir Crit Care Med 1999;160:1254-62.

24. Crain EF, Kercsmar C, Weiss KB, Mitchell H, Lynn H. Reported difficulties in access to quality care for children with asthma in the inner city. Arch Pediatr Adolesc Med 1998;152:333-9.

25. Pohunek P, Spicak V. Current situation of bronchial asthma in the Czech Republic. Allergy \& Clinical Immunology International 2001;13:54-9.

Endereço para correspondência:

Dr. Paulo A.M. Camargos

Dep. de Pediatria - Faculdade de Medicina da UFMG

Avenida Alfredo Balena, 190 - Sala 4061

CEP 30130-100 - Belo Horizonte, MG

Tel.: + 55313248.9773 - Fax: +55 313248.9664

E-mail: pcamargs@medicina.ufmg.br 